\title{
The Management of Complications Related to Hydatid Cysts: Case Report
}

\author{
T. Mesbahi, Y. Tahrir, M. Makhchoune, K. Ibahiouin, and A. Lakhdar
}

\section{ABSTRACT}

This paper presents the case of a 13-year-old female patient, operated in 2015 for left temporoparietal hydatid cyst and reoperated in 2016 for recurrence of the cerebral hydatid cyst. At admission, his GCS scores were E3V4M5. Neurological examination revealed a hemilatéral motor deficit estimated at 3/5 on right side without sensitive trouble. The CT scan showed a large left temporal cystic formation with an infected appearance associated with 4 cystic formations with calcified walls exerting a mass effect on the adjacent parechyma and the homolateral lateral ventricle responsible for a triventricular hydrocephalus. No primary focus was found in the lungs, liver, or other organs. In this article, we will discuss the management of complications related to hydatid cysts. Cerebral hydatid cyst is a rare condition, affecting mainly children. The diagnosis of cerebral hydatid cyst must be evoked in endemic countries in front of a symptomatology of intra-cranial hypertension.

Keywords: Brain, complications, echinococcus, hydatid cyst, prevention.
Submitted: August 7, 2021

Published: October 30, 2020

ISSN: $2593-8339$

DOI: $10.24018 /$ ejmed.2021.3.4.1006

\section{T. Mesbahi}

Centre Hospitalier Universitaire IBN ROCHD Casablanca, Morocco. (e-mail: tarek.mes@gmail.com) Y. Tahrir

Centre Hospitalier Universitaire IBN ROCHD Casablanca, Morocco. (e-mail: yass.med9@gmail.com)

\section{Makhchoune}

Centre Hospitalier Universitaire IBN ROCHD Casablanca, Morocco. (e-mail: makh.mar@gmail.com) K. Ibahiouin

Centre Hospitalier Universitaire IBN ROCHD Casablanca, Morocco. (e-mail: k.ibahouin1@gmail.com) A. Lakhdar Centre Hospitalier Universitaire IBN ROCHD Casablanca, Morocco. (e-mail:

abdelhakiim.lakhdar@gmail.com)

*Corresponding Author

\section{INTRODUCTION}

Hydatid disease is very common in Morocco and the Mediterranean region [1]. Cerebral localization is rare, accounting for only about $2 \%$ of all hydatid localizations in the body, and mainly affects children and adolescents [1]. The clinical signs are those of an intracerebral process, the CT scan is often sufficient to make the diagnosis, and the treatment is surgical [2]. The prognosis is good thanks to improved diagnostic and therapeutic means, the postoperative recurrence is pejorative.

\section{CASE REPORT}

A 13-year-old female patient, right-handed, living in rural 
region, notion of contact with dogs, operated in 2015 for left temporoparietal hydatid cyst and reoperated in 2016 for recurrence of the cerebral hydatid cyst. He was brought by his mother to our emergency for intracranial hypertension syndrome with disorders of consciousness. At admission, his GCS scores were E3V4M5. Neurological examination revealed muscle power was $3 / 5$ on right side, $5 / 5$ on left side without sensitive trouble. The CT scan showed a large left temporal cystic formation with an infected appearance associated with 4 cystic formations with calcified walls exerting a mass effect on the adjacent parechyma and the homolateral lateral ventricle responsible for a triventricular hydrocephalus.

The patient was taken urgently to the operating room. We performed a large craniectomy and then a cortectomy. Then we inserted a flexible probe between the cyst and the cerebral parenchyma to perform the hydropulsion according to the Arana-Iniguez technique. and hypertonic saline is injected.

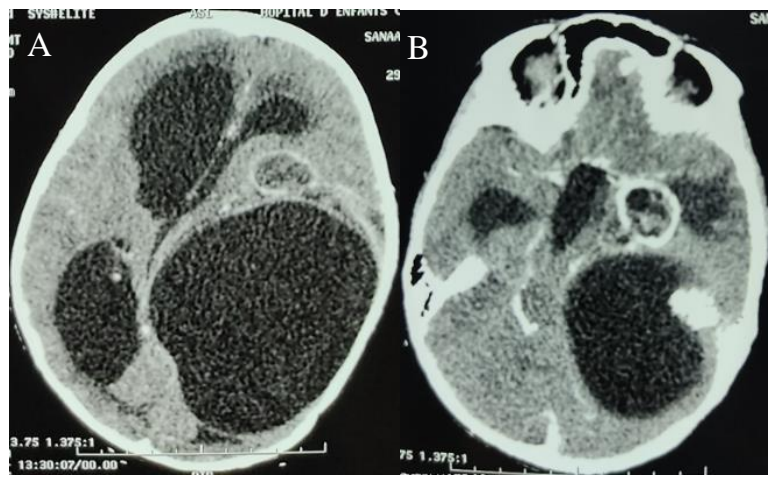

Fig. $1 \mathrm{~A}$ and B. A brain CT scan was performed, showing a round hypodense intra-parenchymal lesion at the left temporal level with an important mass effect on the midline and triventricular hydrocephalus.

The cyst was removed without any incident. We visualised a cavity full of infective cysts which we evacuated and 4 other calcified cysts that we did not touch. On the follow-up, the patient remained somnolent. The cerebral CT scan control showed persistent hydrocephalus and annular contrast in the cavity of the evacuated temporal hydatid cyst.

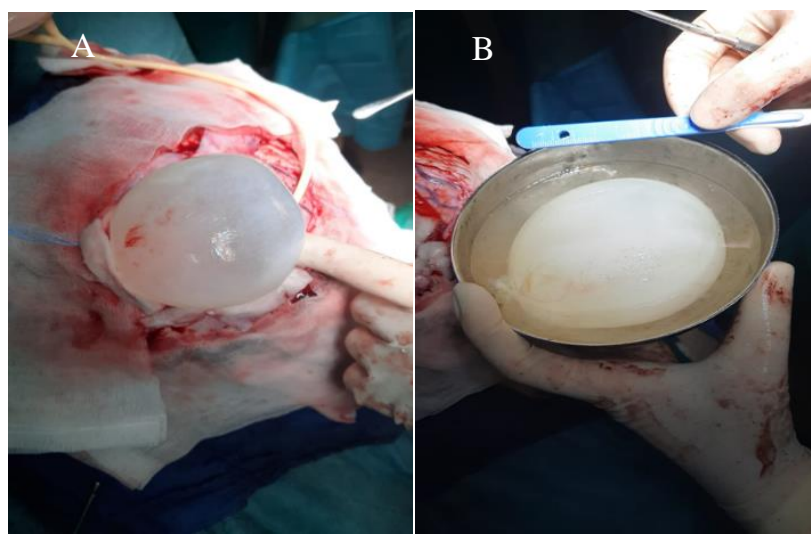

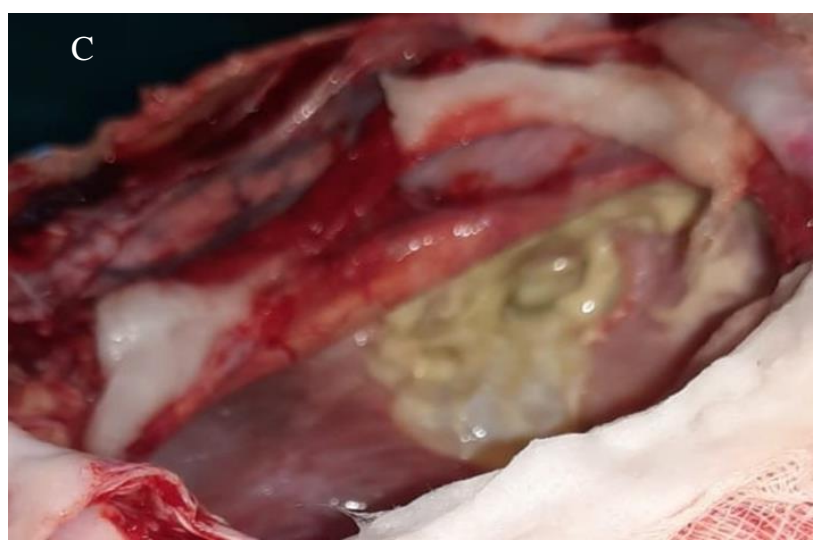

Fig. 2. Preoperative images.

(A) an intraoperative image showing the delivery of the hydatid cyst, (B) image showing the complete removal of the hydatid cyst without its rupture, (C) cavity full of infective cysts.

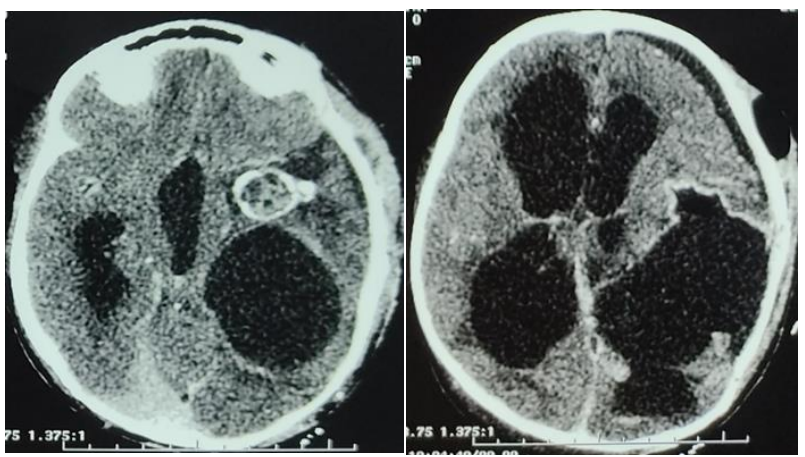

Fig. 3.The control CT scan showing the total removal of the hydatid cyst and persistent hydrocephalus and annular contrast in the cavity of the evacuated temporal hydatid cyst.

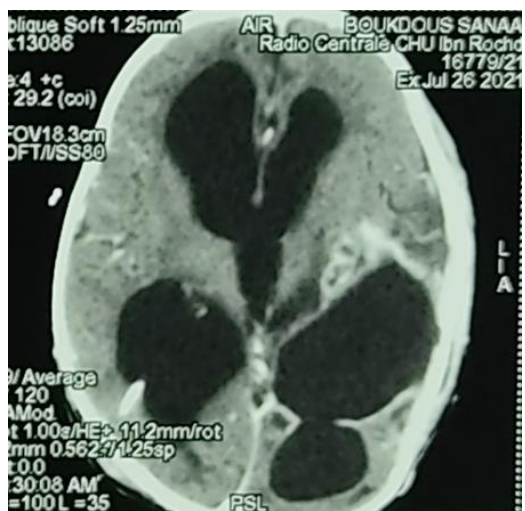

Fig. 4. The control CT scan showing a regression of the hydrocephalus with annular classification of the temporal cyst cavity.

We placed an external ventricular shunt. The patient received 21 days of antibiotics. The cerebrospinal fluid was sterile, so we decided to place an internal ventricular shunt after 3 weeks. Patient's consciousness level was gradually improved. The CT-scan control showed a regression of the hydrocephalus with annular classification of the temporal cyst cavity. She was discharged 3 days later.

\section{DISCUSSION}

Hydatidosis is a cosmopolitan parasitosis that is still endemic in our country, constituting a real public health problem. Usually observed in the liver $(60 \%)$ and the lungs (30\%), encephalic localization remains rare, not exceeding 
$2 \%$ of all somatic localizations. Children are the main victims $(50-75 \%)$ given the frequency of contact with dogs and the poor hygiene conditions at this age, particularly in rural areas. The average age is 5 to 8 years [2].

Intracranial hypertension is almost constant [4], it expresses the presence of an expansive intracranial process which settles in progressively over several weeks or even several months, due to the slow growth of the cyst and the relative elasticity of the child's cranial cavity: The headaches are progressive and of increasing intensity, with morning vomiting relieving the headaches. Visual disturbances are sometimes difficult to detect in young children, but uni or bilateral papillary oedema is often found during the ophthalmological examination [2], [3]. Other signs may accompany or reveal the hydatid cyst in the brain: neurological deficits such as paresis, or rarely paralysis [5]; generalized tonic-clonic seizures, especially in remodeled forms [6]; behavioral or psychiatric disorders may be present, or even disturbances of consciousness ranging from obnubilation to true coma [7].

The diagnostic means are represented by brain CT and MRI $[1,8]$. Imaging helps the surgeon to plan the operation. The hydatid cyst is often a unique lesion, intraparenchymal, supratentorial, well limited, often spherical, with a density identical to that of the cerebrospinal fluid, its wall is thin and is not enhanced by the contrast product, there is no perilesional oedema. Imaging also makes it possible to assess the size of the cyst and thus measure the risk of intraoperative rupture [11].

The MRI is the examination of choice, showing a lesion in hypo-signal T1, hyper-signal T2 also better at detecting multiple hydatid cerebral cysts and better defines the anatomy of the lesion to the surrounding structures, which helps in surgical planning, but the CT scan is superior in the detection of calcifications [11].

Brain MRI may be essential in cases of remodeled cysts and will help to eliminate differential diagnoses. The problems of differential diagnosis may arise especially in cases of cyst remodeling; these differential diagnoses are cystic gliomas, arachnoid cysts, other infectious processes; the cyst wall is thick in these cases with or without contrast. Cerebral MRI is better at localization and characterization of cerebral hydatid cysts than CT [7]. Other locations may be associated in approximately $30 \%$ of cases [3], particularly liver and lung, and these should be systematically sought.

Biological tests are of limited diagnostic value. Hydatid serology is positive in $70 \%$ of cases of hepatic cysts, but only $20 \%$ of isolated cerebral hydatid cysts. The diagnosis is histological, and it reveals germenative membrane of the cyst. [11].

The treatment of cerebral hydatid cysts is both medical and surgical. Surgical treatment should be considered whenever possible. The Arana-Iniguez hydraulic enucleation technique separates the $\mathrm{KH}$ from the brain parenchyma and delivers it progressively by instilling saline through a flexible tube inserted between the parenchyma and the cyst [6]. This method is simple and only poses a problem if the cyst is remodeled or located in deep regions, especially the brainstem, or narrow regions such as the posterior cerebral fossa, because the volume added during hydrodissection can worsen the compression of the brainstem intraoperatively [1]. In these cases, and despite the major risk of rupture and brain seeding, the Digaammaimaginario puncture-aspiration method should be used [6]; it consists of puncturing and aspirating the contents of the cyst before proceeding with mass removal of the residual membrane. [11].

Medical treatment with albendazol is indicated in case of rupture, or in case of multiple locations. The results of drug treatment of hydatid cysts remain variable depending on the series, with response rates ranging from 43.5 to $80 \%$ [11].

The particularity of our observation is the management of complications of hydatid cysts, such as infection and hydrocephalus. The patient was neurologically confused after the first surgery. The control CT scan showed active hydrocephalus despite the removal of the $\mathrm{KH}$. We explained the hydrocephalus by the disturbance of the hemodynamics of the circulation of the cerebrospinal fluid.

We administered antibiotics after we found the infected cavity intraoperatively. we suppose that the appearance of calcification of the evacuated temporal hydatid cyst cavity is a sign of healing.

The prognosis is generally good if diagnosed early and apart from multiple brain locations which pose a serious therapeutic problem [11].

\section{CONCLUSION}

Cerebral hydatid cyst is a rare condition, affecting mainly children. The diagnosis of cerebral hydatid cyst must be evoked in endemic countries in front of a symptomatology of intra-cranial hypertension. The diagnosis is easily made by CT scan. The treatment is medical-surgical, and the prognosis is generally good. However, health education, control of the agent of transmission of the parasite (stray dogs) and meat inspection remains the only means to eradicate this pathology.

\section{REFERENCES}

[1] N. Yilmaz, N. Kiymaz, O. Etlik, T. Yazici. "Primary Hydatid Cyst of the Brain During Pregnancy," NeurolMedChir, 46(8):415-7, 2006.

[2] R. A. Agha, T. Franchi, C. Sohrabi, G. Mathew, for the SCARE Group. The SCARE 2020 Guideline: Updating Consensus Surgical CAse REport (SCARE) Guidelines, International Journal of Surgery, 84:226-230, 2020.

[3] M. Brahem, K. Hlel, A. Ayadi, A. Bedoui, F. Hmila, B. Mahjoub, ... and M. T. Sfar, "Cerebral hydatid cysts in children: 4 cases," Medecine et maladies infectieuses, 36(8), 434-437, 2006.

[4] K. Demir, A. F. Karsli, T. Kaya, E. Devrimci, K. Alkan, "Cerebral hydatid cysts: CT findings,” Neuroradiology, 33: 22-24, 1991.

[5] C. A. Gonzalez-Ruiz, A. Isla, A. Perez-Higueras, M. G. Blazquez, "Unusual CT image of a cerebral hydatid cyst," Pediatr Radiol, 20: 283-284, 1990.

[6] D. R. Reddy, J. M. K. Murthy, "Parasitic intracranial space- occupying lesions in children in India," Childs Nerv Syst, 2: 244-247, 1986.

[7] E. W. Braunsdorf, D. Schmidt, M. Rautenberg, "Cerebral manifestation of hydatid disease in a child," Childs Nerv Syst, 4: 249-251, 1988.

[8] T. Golaszewski, M. Susani, S. Golaszewski, G. Sliutz, G. Bischof, H. Auer, "A large hydatid cyst of the liver pregnancy," Arch Gynecol Obstet, 256: 43-47, 1995.

[9] R. Krajewski, Z. Stelmasiak, "Cerebral hydatid cysts in children," Childs Nerv Syst, 7: 154-155, 1991. 
[10] J. Handa, Y. Nakano, H. Handa, "Computed tomography in the differential diagnosis of low- density intracranial lesions," Surg Neurol, 10: 179-185, 1978 .

[11] K. Khattala, A. Elmadi, M. Rami, A. Mahmoudi, Y. Bouabdallah, "Huge cerebral hydatid cyst revealed by a coma," The Pan African Medical Journal, 13, 64-64, 2012.

[12] M. Melis, L. Marongiu, F. Scintu, M. Pisano, F. Capra, L. Zorcolo, G. Casula, "Primary hydatid cysts of psoas muscle," ANZ J Surg, 72: 443445, 2002.

[13] J. Vaquero, C. Jimenez, R. Mantirez, "Growth of hydatid embolism," Case report. J Neurosurg, 57: 837-838, 1982 